Full-length article

\title{
Development of efficient RNA interference system using EGF-displaying phagemid particles
}

\author{
Hua JIANG ${ }^{2,4}$, Xiu-mei CAI ${ }^{3,4}$, Bi-zhi SHI ${ }^{2}$, Jie ZHANG ${ }^{2}$, Zong-hai LI $^{2,5}$, Jian-ren GU²,3 \\ ${ }^{2}$ State Key Laboratory for Oncogenes and Related Genes, Shanghai Jiao Tong University, Shanghai 200032, China; ${ }^{3}$ Medical College of Fudan \\ University, Shanghai 200031, China
}

\author{
Key words \\ epidermal growth factor; siRNA; gene delivery; \\ phagemid particles; hydroxycamptothecin \\ ${ }^{1}$ Project supported by the National 973 Basic \\ Research Program of China (№ 2004CB518802) \\ and the Science and Technology Commission \\ of Shanghai Municipality (№ 054119568). \\ ${ }^{4}$ These authors contributed equally to this work. \\ ${ }^{5}$ Correspondence to Associate Prof Zong-hai \\ LI. \\ Phn/Fax 86-21-6443-2027. \\ E-mail Zonghaili@gmail.com \\ Received 2007-10-15 \\ Accepted 2007-11-26 \\ doi: $10.1111 / \mathrm{j} .1745-7254.2008 .00768 . \mathrm{x}$
}

\begin{abstract}
Aim: To develop an efficient RNA interference system using phagemid particles displaying the epidermal growth factor (EGF) ligand. Methods: pSilencer1.0-siEGFP and pSilencer4.1-siAkt plasmids were constructed by gene clone technology. The modified helper phage genome (plasmid) M13KO7EGFCT was used to package phagemids, such as pSilencer 1.0-siEGFP and pSilencer4.1-siAkt. ELISA was used to quantify the titer of the progeny virus particles. Single-strand DNA was extracted and analyzed by agarose gel electrophoresis to evaluate the percentage of the phagemid particles. The expression level of the reporter gene enhanced green fluorescence protein (EGFP) was determined by transducing phagemid particles packaging pSilencer1.0-siEGFP into cells. The level of Akt gene expression in cells transduced phagemid particles packaging pSilencer4.1-siAkt was examined by Western blotting. Hydroxycamptothecin (HCPT) was used to enhance the gene transduction efficiency. Results: RNAi vectors pSilencer1.0-siEGFP and pSilencer4.1-siAkt were successfully constructed. Phagemid-encoding siRNA can be packaged efficiently. After the cells were infected by EGF displaying phagemid particles in the presence of HCPT, the expression of the target gene EGFP or Akt was substantially downregulated. Conclusion: Cell-targeted phagemid particles are efficient siRNA delivery vectors in the presence of HCPT.
\end{abstract}

\section{Introduction}

In previous studies, scientists have successfully modified the filamentous bacteriophages, which are capable of delivering genes to mammalian cells using targeting ligands, such as growth factors ${ }^{[1-5]}$, antibodies ${ }^{[6]}$, and viral capsid proteins $^{[7]}$. To improve the ligand display level on phages, we developed LMP cells (Escherichia coli bearing the ligandpIII-encoding helper phage) to make cell-targeted phagemid particles ${ }^{[8]}$. We also demonstrated that it can deliver reporter genes into target cells.

RNAi techniques are a promising way to silence therapeutic target genes. Both non-viral vectors and viral vectors have been used as a vehicle to deliver siRNA in vitro and in vivo. The major problem of non-viral vectors lies in its low gene delivery efficiency and hard quality control. Animal viruses, such as adenoviruses, have broad natural tro- pism which makes them difficult to selectively transfer siRNA genes into target cells. In contrast to these animal viruses, filamentous bacteriophage has no natural tropism to mammalian cells. However, it can highly efficiently infect the targeted cells when the peptide ligand was displayed on the surface. Like non-viral vectors, the major problem of this vector is its low gene delivery efficiency. Fortunately, hydroxycamptothecin (HCPT), a topoisomerase I inhibitor and also anticancer drug, can substantially improve its gene delivery efficiency ${ }^{[1,13,17,18]}$. In this study, we tested whether cell-targeted phages can be used as siRNA gene delivery tools or not.

\section{Materials and methods}

Reagents Restriction endonucleases were purchased from TaKaRa Biotechnology (Dalian, China). Dulbecco's 
modified Eagle's medium (DMEM), Dulbecco's phosphatebuffered saline, fetal bovine serum (FBS), and trypsin/ethylenediamine tetraacetic acid were purchased from Invitrogen (Grand Island, NY, USA).

Cell culture H1299 (large-cell lung carcinoma; ATCC, Manassas, VA, USA) cells were cultured at $37^{\circ} \mathrm{C}$ in medium consisting of $90 \%$ DMEM and $10 \%$ FBS in a humidified atmosphere of $95 \%$ air and $5 \% \mathrm{CO}_{2}$.

Plasmid construction All oligonucleotides were synthesized by Sangon (Shanghai, China) and annealed to be double-stranded, as previously described ${ }^{[10]}$. The selection of the coding sequences of EGFP and Akt for siRNA was based on 2 different publications, respectively ${ }^{[9,10]}$. Detailed information of the oligonucleotides is shown in Table 1.

To generate the pSi1.0-EGFP RNAi plasmid, a 55-nt oligoDNA duplex (annealed by siEGFP-f and siEGFP-r) corresponding to nucleotides 106-127 of the pEGFP-N1 (BD Biosciences Clontech, Palo Alto, CA, USA)-coding region was directly inserted into the pSilencer1.0 (Ambion, Austin, TX, USA) vector digested with ApaI (blunted) and HindIII.

The pSilencer4.0-f plasmid was generated as follows: the pGl3-control plasmid (Promega categoryno.: E1741; Promega, Madison, WI,USA) was digested with $S c a \mathrm{I}$, and the $1.08 \mathrm{~kb}$ fragment were used to ligate with pSilencer4.0 (Ambion, Austin, TX, USA) which is linearized with ScaI. The inserted subclones with the reversed orientation of cytomegalovirus (CMV) promoter are genetically selected with the supplementary of ampicillin resistance. This modified psilencer4.0 was prepared for the phage package and siRNA expression.

To generate the pSi4.0-Akt plasmid, a 52 nt oligo-DNA duplex (annealed by siAkt-f and siAkt-r) corresponding to nucleotides 1462-1482 of the Akt1 (NM_001014432) and 1244-1264 of Akt2 (NM_001626)-coding region was directly inserted into the pSilencer4.0 vector digested with BamHI and HindIII. The mock Akt sequence is only one nucleotide different from siAkt, as listed in Table 1.

Preparation of phagemid particles The phagemid particles were prepared following previously published protocols $^{[11]}$ with some modifications. M13KO7EGFCT ${ }^{[8]}$ was transformed into Escherichia coli to make LMP cells. The phagemid carrying the siRNA-encoding sequence was transformed into LMP cells. The cells were then plated on LB agar (containing $70 \mu \mathrm{g} / \mathrm{mL}$ kanamycin and $50 \mu \mathrm{g} / \mathrm{mL}$ ampicillin) and incubated at $37^{\circ} \mathrm{C}$ overnight, A cell clone was picked the next day and transferred into $1 \mathrm{~L} \mathrm{LB}$ containing $70 \mu \mathrm{g} / \mathrm{mL}$ kanamycin and $50 \mu \mathrm{g} / \mathrm{mL}$ ampicillin. After shaking at $37^{\circ} \mathrm{C}$ for $14-16 \mathrm{~h}$, the supernatant of the culture was collected and the phagemid particles were purified with polyethylene glycol (PEG)/NaCl precipitation.

Quantification of phagemid particles The phagemid particles were quantified by ELISA, as previously described ${ }^{[12]}$. Briefly, serial dilutions of phagemid particles in coating buffer $\left(0.1 \mathrm{~mol} / \mathrm{L} \mathrm{NaHCO}_{3}, \mathrm{pH} 9.1\right)$ were coated in microtiter plates (Corning; Corning, NY, USA) at $4{ }^{\circ} \mathrm{C}$ overnight. After blocking with $1 \%$ bovine serum albumin (BSA; Sigma, St Louis, MO, USA) in phosphate-buffered saline (PBS), the bound phages were stained with a mouse anti-M13 antibody conjugated with horseradish peroxidase (HRP; Amersham Biosciences, Piscataway, NJ, USA) diluted at 1:200 in PBST (PBS containing 0.1\% Tween-20). The signal was developed using the 2,2'-azino-di-(3ethylbenzthiazoline-6-sulfonic acid) substrate and quantitated in an ELISA reader (Bio-Rad Laboratories, Hercules, CA, USA). The M13KO7 helper phage of a known number of pfus was used for standardization.

Table 1. Oligonucleotide sequences used to synthesize siRNA in vitro targeting EGFP and Akt (human) genes.

\begin{tabular}{|c|c|c|c|}
\hline Targets & & Target sequences & Synthesized oligonucleotide \\
\hline \multirow[t]{4}{*}{ EGFP } & siEGFP-f & GGCGATGCCACCTACGGCAAGC & 5'-GGCGATGCCACCTACGGCAAGCTCGAGC \\
\hline & & & TTGCCGTAGGTGGCATCGCCCTTTTTG-3' \\
\hline & siEGFP-r & & 5'-AAT TCAAAAAGGGCGATGCCACCTACGGC \\
\hline & & & AAGCTCGAGCTTGCCGTAGGTGGCATCGCC-3' \\
\hline \multirow[t]{8}{*}{$\mathrm{Akt}$} & siAkt-f & CCTGCCCTTCTACAACCAGGA & 5'-GATCCTGCCCTTCTACAACCAGGACTGCA \\
\hline & & & GTCCTGGTTGTAGAAGGGCAGG A-3' \\
\hline & siAkt-r & & 5'-AGCTTCCTGCCCTTCTACAACCAGGACTG \\
\hline & & & CAGTCCTGGTTGTAGAAGGGCA G-3’' \\
\hline & siAktm-f & CCTGCCTTTCTACAACCAGGA & 5'-GATCCTGCCTTTCTACAACCAGGACTGCA \\
\hline & & & GTCCTGGTTGTAGAAAGGCAGG A-3' \\
\hline & siAktm-r & & 5'-AGCTT СCTGCCTTTCTACAACCAGGA \\
\hline & & & CTGCAG TCCTGGTTGTAGAAAGGCA G-3' \\
\hline
\end{tabular}


Purification of single-strand DNA from phagemid particles Single-strand DNA (ssDNA) was extracted from the phagemid particles following the supplied protocol (Ph.D.12 phage display peptide library kit, New England Biolabs, Beverly, MA, USA). Briefly, the phagemid particles were precipitated by $\mathrm{PEG} / \mathrm{NaCl}$. The pellet was then suspended with $100 \mu \mathrm{L}$ iodide buffer. In total, $250 \mathrm{~mL}$ ethanol was added and incubated for $10 \mathrm{~min}$ to allow for SsDNA precipitation. The pellet was collected after centrifugation at $12000 \times g$ for $10 \mathrm{~min}$, washed in $70 \%$ ethanol, and air dried for $10 \mathrm{~min}$. It was finally dissolved in $30 \mu \mathrm{L}$ TE buffer $(10 \mathrm{mmol} / \mathrm{L}$ Tris- $\mathrm{HCl}$ [pH 8.0] and $1 \mathrm{mmol} / \mathrm{L}$ EDTA) and analyzed by agarose gel electrophoresis.

In vitro phagemid particle transfection The cells were plated into 24-well plates at densities of 10000 cells per well, $24 \mathrm{~h}$ prior to the phage particle addition. The phages were added at $10^{11} \mathrm{pfu} / \mathrm{mL}$ and incubated with the cells for $48 \mathrm{~h}$ at $37^{\circ} \mathrm{C}$ in complete media. The cells were visualized under an epifluorescent inverted microscope (IX71; Olympus, Tokyo, Japan). All the transfections were done in triplicate and performed at least twice.

HCPT treatment HCPT treatments were performed $48 \mathrm{~h}$ after the addition of the phages in medium containing $10 \%$ FBS. The medium was removed at $48 \mathrm{~h}$ after the phage addition, and the cells were incubated with $\mathrm{HCPT}$ at $2.5 \mu \mathrm{mol} / \mathrm{L}$ for $6 \mathrm{~h}$ at $37^{\circ} \mathrm{C}$. This was followed by replacement with fresh medium and an additional incubation of $18 \mathrm{~h}$ at $37^{\circ} \mathrm{C}$.

Western blot analysis In total, $10^{6} \mathrm{H} 1299$ cells transfected transiently with a variety of phagemid particles were lysed after HCPT treatment, as described in the Results. Prior to the lyses, the cells were washed with PBS and collected by scraping. Then, they were lysed in ice-cold Tris buffer (50 mmol/L, pH 7.5) containing $5 \mathrm{mmol} / \mathrm{LEDTA}, 300 \mathrm{mmol} / \mathrm{L}$ $\mathrm{NaCl}, 0.1 \%$ Igepal, $0.5 \mathrm{mmol} / \mathrm{L} \mathrm{NaF}, 0.5 \mathrm{mmol} / \mathrm{L} \mathrm{Na}_{3} \mathrm{VO}_{4}, 0.5$ $\mathrm{mmol} / \mathrm{L}$ phenylmethylsulfonyl fluoride, and antiprotease mixture (Roche Molecular Biochemicals, Indianapolis, IN, USA), sonicated, and centrifuged at $13000 \times g$ for $10 \mathrm{~min}$. The supernatant was used for protein determination by the Bradford procedure (Bio-Rad, USA) and Western blotting. The proteins were resolved on $12 \%$ SDS-PAGE, transferred onto nitrocellulose membranes, and incubated with the appropriate antibodies. The following protocols are the same as described earlier. The anti-pSer473-AKT antibody (Cell Signaling Technology, Beverly, MA, USA) was used in 1:1000 dilutions. The goat antimouse immunoglobulin $\mathrm{G}$ conjugated with HRP (ImmuClub Labs, Sunnyvale, CA, USA) was diluted at 1:10 000 in 1\% BSA in PBST. After $1 \mathrm{~h}$ incubation, the filters were washed with PBST, developed using a SuperSignal West Pico kit (Pierce Biotechnologies, Rockford,
IL, USA) for $5 \mathrm{~min}$, and exposed to X-ray film.

\section{Results}

Construction of siRNA expression vectors To improve the expression of siRNA against Akt, the pSilencer4.1-CMV vector which carries the CMV promoter was used. pSi4.1CMVfl was constructed by inserting the F1 origin sequence into pSilencer4.1-CMV. pSilencer4.0-siAKT was obtained by insert shRNA against Akt into pSi4.1CMV-fl.

ssDNA analysis To examine the ratio of phagemids to helper phage genomes packaged in the phagemid particles, we analyzed the ssDNA. The results indicate that almost all of the DNA packaged were phagemids (Figure 1).

In vitro inhibition of EGFP expression by EGF displaying pSil.0-siEGFP phagemid particle infection To test whether phagemid particles carrying siRNA against EGFP can inhibit EGFP expression, we transfected the pEGFP-N1 plasmid into NCI-H1299 cells. At $12 \mathrm{~h}$ after transfection, the phagemid particles $\left(10^{11} \mathrm{pfu} / \mathrm{mL}\right)$ were transduced into the cells. At $24 \mathrm{~h}$ after transduction, HCPT was added into the culture at a final concentration of $2.5 \mu \mathrm{mol} / \mathrm{L}$; the cells was incubated for another $24 \mathrm{~h}$. The data shown in Figure 2 indicate that pSil.0-siEGFP can significantly inhibit the expression of EGFP.

Phagemid particles of pSi4.1-siAkt can inhibit Akt expression of NCI-H1299 in the presence of HCPT The NCIH1299 cells was infected with pSi4.1-siAkt phagemid particles packaged by M13KO7EGFCT. HCPT was added to improve the siRNA expression. Western blotting was performed to examine the Akt expression.

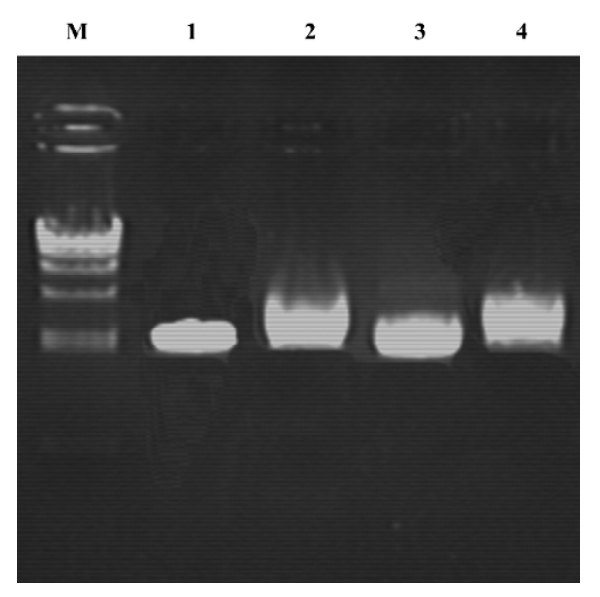

Figure 1. Analysis of the ssDNA released from phagemid particles Lane M: $\lambda$ DNA/HindIII; Lane 1: pSi1.0-siEGFP phagemid particles; Lane 2: pSi4.1-siAkt phagemid particles; Lane 3: pSi1.0-Mock phagemid particles; Lane 4: pSi4.1-Mock phagemid particles 
A
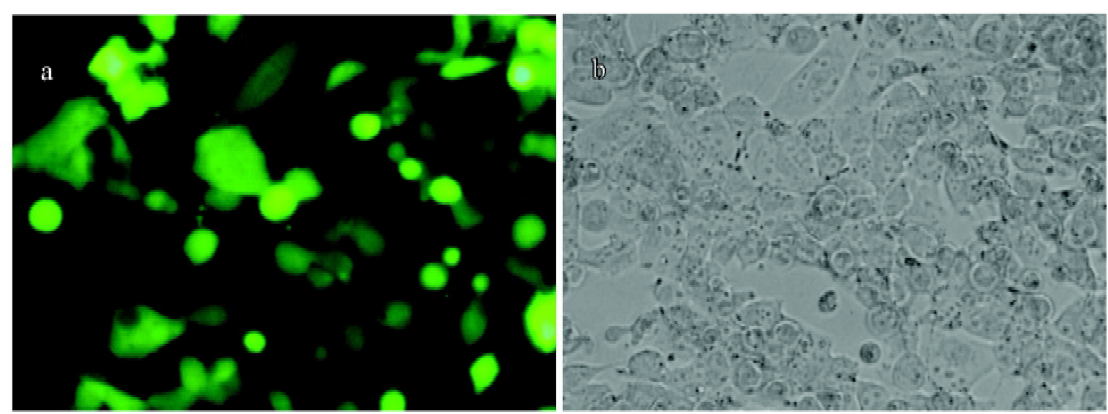

B
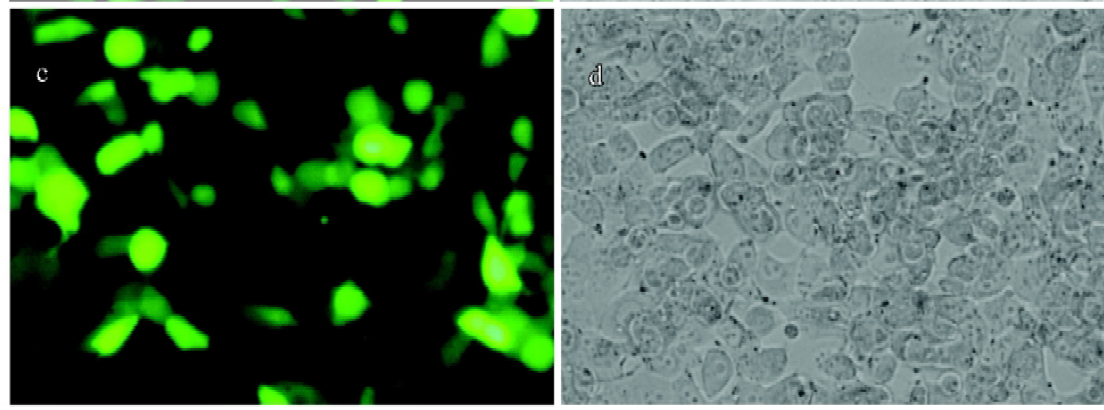

C
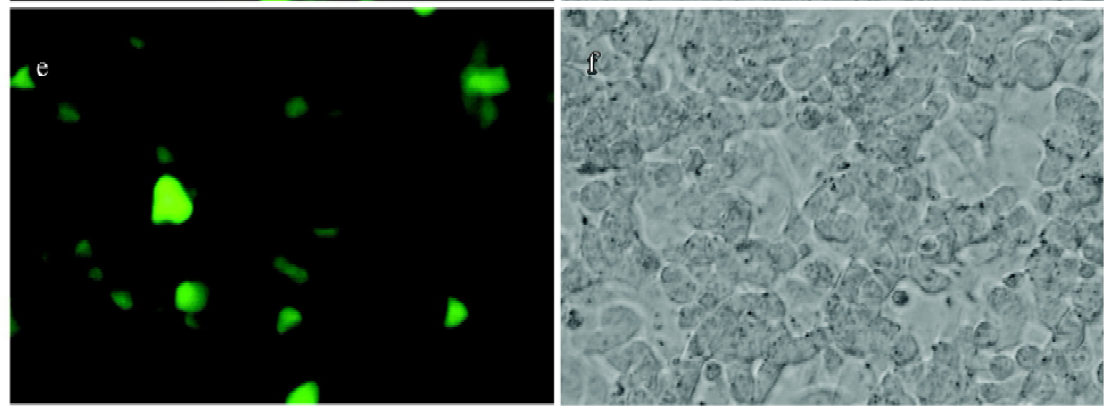

D

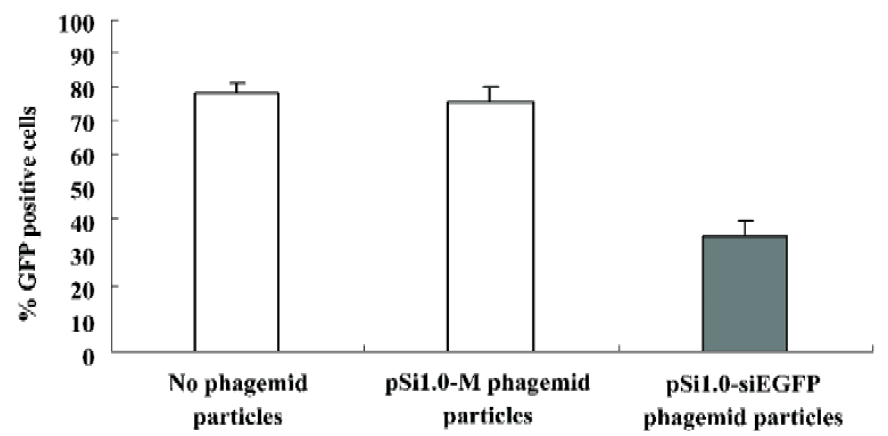

Figure 2 Inhibiting EGFP expression by transduction of pSil.0- siEGFP phagemid particles into NCI-H1299 cells carrying pEGFP-N1 in the presence of hydroxycamptothecin. (A) No phagemid particles added; (B) pSi1.0-M phagemid particles packaged with M13EGFKO7CT; (C) pSil.0-siEGFP phagemid particles packaged with M13EGFKO7CT; (D) Quantitation of GFP positive cells transfected by phagemid particles (GFP positive cells from duplicate wells were averaged and experiments were performed in triplicate. In total, 50000 cells per well were counted each time. Error bars represent the standard deviation of the mean). All the images were taken at the same exposure. (Magnification: 400). a,c,e, represent cells visualized under fluorescence microscope; $b$, d, f, represent cells visualized under light microscope.

As shown in Figure 3A, the pSi4.1-siAKT plasmid transfected by Lipofectamine can inhibit the Akt expression by approximately $50 \%$. No inhibition was found in mock vector
pSi4.1-M.

As shown in Figure 3B, in the absence of HCPT, the phagemid particles of siAkt can not efficiently inhibit the 
A

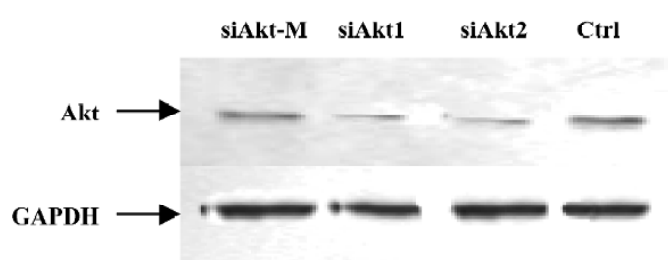

B

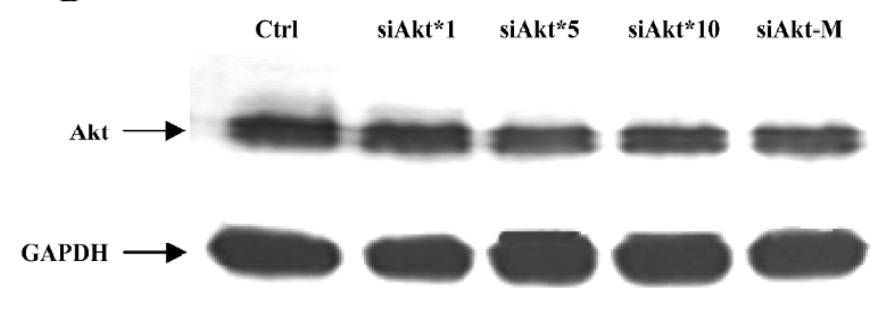

C

Ctrl siAkt*1 siAkt*5 siAkt*10 siAkt-M

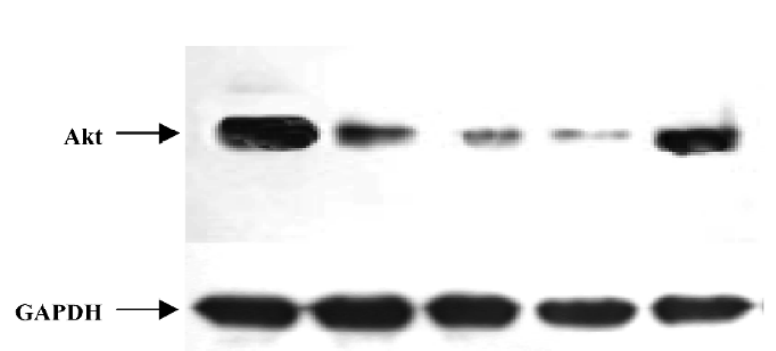

Akt expression. However, in the presence of HCPT (Figure 3C), the siAKT phagemid particles can inhibit the Akt expression in a dose-dependent manner. The highest inhibition was $50 \%-60 \%$ when $1 \times 10^{11} \mathrm{pfu} / \mathrm{mL}$ particles were added.

\section{Discussion}

In this study, we utilized a novel phagemid system to deliver several siRNA into mammalian cells and estimated their effect in vitro. The phagemid vectors have a number of advantages over other vectors. Unlike other viral gene delivery vector, filamentous phages have no natural mammalian cell tropism. Phagemid particles can be easily retargeted by displaying various ligands, such as peptides, scFv and natural ligands (eg EGF, FGF $)^{[5,8,14-16]}$. In addition, it is very efficient to prepare the phagemid particles rapidly (about 2$3 \mathrm{~d}$ ). In previous studies, phages were modified so that they could be used in gene delivery. The ability of cell-targeted
Figure 3 Western-blot analysis of the specific Akt gene silencing by EGF-targeted phagemid particles mediated RNA interference.

(A) pSi4.1-siAkt RNAi expression plasmid was transfected into NCIH1299 cells using Lipofectamine 2000. 72 h later the cells were harvested, and the obtained total cell lysates were subjected to SDSPAGE and immunoblotting; siAkt1 and siAkt2 are duplicate experiments of the siAkt transfection. (B) NCI-H1299 cells were transfected with different doses of pSi4.1- siAkt phagemid particles in the absence of hydroxycamptothecin; (C) NCI-H1299 cells were transfected with different doses of pSi4.1-siAkt phagemid particles in the presence of $2.5 \mu \mathrm{mol} / \mathrm{L}$ hydroxycamptothecin. siAkt ${ }^{*} 1$, siAkt 5 and siAkt ${ }^{*} 10$ represent $1 \times 10^{10} \mathrm{pfu} / \mathrm{mL}, 5 \times 10^{10} \mathrm{pfu} / \mathrm{mL}, 1 \times 10^{11} \mathrm{pfu} / \mathrm{mL} \mathrm{pSi} 4.1$-siAkt phagemid particles respectively. $\mathrm{Ctrl}$ represent no phagemid particles added. siAkt-M represent mock siRNA phagemid particles.

phages to deliver siRNA would be of interest. Since phage genomes for packaging replicates by rolling circles, we examined in this study whether the hairpin structure of the siRNA-encoding sequence will impede its replication and package. The results clearly demonstrate that phagemid particles carrying siRNA can be prepared as efficiently as those carrying other protein-encoding genes.

This study also demonstrates that cell-targeted phagemid particles carrying siRNA can be expressed efficiently in the presence of HCPT, although further studies need to be performed to elucidate the mechanism.

We also tested whether the phagemid particles carrying siRNA against Akt have some effect on cell growth. However, no significant inhibition was obtained. This is not uncommon, because the EGF ligand on the phagemid particles has a cell-growth stimulatory effect that might compensate the cell growth inhibitory effect contributed by the siRNA. Thus, to be a cancer gene-delivery vector, phage vectors should display ligands that do not prompt cell growth 
or carry siRNA against other oncogenes, such as PI3K.

\section{References}

1 Burg MA, Jensen-Pergakes K, Gonzalez AM, Ravey P, Baird A, Larocca D. Enhanced phagemid particle gene transfer in camptothecin-treated carcinoma cells. Cancer Res 2002; 62: 977-81.

2 Larocca D, Witte A, Johnson W, Pierce GF, Baird A. Targeting bacteriophage to mammalian cell surface receptors for gene delivery. Hum Gene Ther 1998; 9: 2393-9.

3 Larocca D, Kassner PD, Witte A, Ladner RC, Pierce GF, Baird A. Gene transfer to mammalian cells using genetically targeted filamentous bacteriophage. FASEB J 1999; 13: 727-34.

4 Kassner PD, Burg MA, Baird A, Larocca D. Genetic selection of phage engineered for receptor-mediated gene transfer to mammalian cells. Biochem Biophys Res Commun 1999; 264: 9218.

5 Li ZH, Zhang J, Zhao RJ, Xu YH, Gu JR. Preparation of peptidetargeted phagemid particles using a protein III-modified helper phage. Biotechniques 2005; 39: 493-7.

6 Poul MA, Marks JD. Targeted gene delivery to mammalian cells by filamentous bacteriophage. J Mol Biol 1999; 288: 203-11.

7 Di Giovine M, Salone B, Martina Y, Amati V, Zambruno G, Cundari E, et al. Cell delivery and gene transfer of adenoviral penton base displaying bacteriophage. Virology 2001; 282: 10212.

8 Li ZH, Jiang H, Zhang J, Shi BZ, Gu JR. Cell targeted phagemid particles preparation using Ecoli bearing ligand-pIII encoding helper phage genome. BioTechniques 2006; 41: 706-7.

9 Sui G, Soohoo C, Affar el B, Gay F, Shi Y, Forrester WC, et al. A DNA vector-based RNAi technology to suppress gene expression in mammalian cells. Proc Natl Acad Sci USA 2002; 99: 551520 .

10 Katome T, Obata T, Matsushima1 R, Masuyama N, Cantley LC, Gotoh Y, et al. Use of RNA interference-mediated gene silencing and adenoviral overexpression to elucidate the roles of AKT/ protein kinase B isoforms in insulin actions. J Biol Chem 2003; 278: 28312-23.

11 Barbas CF. Phage display: a laboratory manual. CSH Laboratory Press; 2001.

12 Rondot S, Koch J, Breitling F, Dübel S. A helper phage to improve single-chain antibody presentation in pahge display. Nat Biotechnol 2001; 19: 75-78.

13 Alexander IE, Russell DW, Miller AD. DNA-damaging agents greatly increase the transduction of nondividing cells by adenoassociated virus vectors. J Virol 1994; 68: 8282-7.

14 Gao CS, Mao SL, Lo CL, Wirsching P, Lerner RA, Janda KD. Making artificial antibodies: a format for phage display of combinatorial heterodimeric arrays. Proc Natl Acad Sci USA 1999; 96: 6025-30.

15 Kwas'nikowski P, Kristensen P, Markiewicz WT. Multivalent display system on filamentous bacteriophage pVII minor coat protein. J Immunol Methods 2005; 307: 135-43.

16 Gao C, Mao S, Ditzel HJ, Farnaes L, Wirsching P, Lerner RA, et al. A cell-penetrating peptide from a novel pVII-pIX phagedisplayed random peptide library. Bioorg Med Chem 2002; 10 : 4057-65.

17 Zhang R, Li Y, Cai Q, Liu T, Sun H, Chambless B, et al. Preclinical pharmacology of the nature product anticancer agent 10hydroxycamptothecin, an inhibitor or topoisomerase I. Cancer Chemother Pharmacol 1998; 41: 257-67.

18 Zhou JJ, Liu J, Xu B. Relation between lactone ring forms of HCPT and their antitumor activities. Acta Pharmacol Sin 2001; 22: $827-30$. 